\title{
Localization of Bounded Sets in Tensor Products
}

\author{
A. PERIS and M.J. RIVERA
}

\begin{abstract}
The problem of topologies of Grothendieck is considered for complete tensor products of Fréchet spaces endowed with the topology defined by an arbitrary tensor norm. Some consequences on the stability of certain locally convex properties in spaces of operators are also given.
\end{abstract}

Grothendieck, in his work about Fréchet and (DF)-spaces and tensor products (see [12], [13]), studied locally convex properties of function spaces. Many examples of spaces of vector-valued functions can be represented as tensor products and this motivated the study of the topological structure of tensor products of Fréchet and (DF)-spaces.

The "probléme des topologies" of Grothendieck asks if every bounded set $B$ of the complete projective tensor product $E \hat{\otimes}_{\pi} F$ of two Fréchet spaces $E$ and $F$ can be "localized", i.e. if there are bounded subsets $C, D$ of $E$ and $F$ respectively with $B \subset \overline{\Gamma(C \otimes D)}$. Due to

1991 Mathematics Subject Classification: 46A04, 46A16, 46A32, $46 \mathrm{M05}$.

Servicio publicaciones Univ. Complutense. Madrid, 1996.

The research was partially supported by DGICYT under Proyecto PB91-0538 
the important work of Taskinen (see [22]) we know that the answer is negative in general. Bonet, Díaz and Taskinen [6], inspired by Bonet and Díaz [5] and Taskinen [22,23], introduced the classes of (FG) and (DFG)-spaces as a general frame in which the "probléme des topologies" of Grothendieck (see [13]) and some related dual questions have a positive answer. The purpose of this paper is to extend the results of Bonet, Díaz and Taskinen for arbitrary tensor norms and show that the bounded sets of the $\alpha$-tensor product $E \hat{\otimes}_{\alpha} F$ of two (FG)-spaces $E$ and $F$ can be localized in a "canonical sense". Analogously it is proved that the $\alpha$-tensor product $G \otimes_{\alpha} H$ of two (DFG)-spaces $G$ and $H$ is always a (DF)-space. This also extends the corresponding results for the Lapresté's $\alpha_{p q}$-tensor products due to López Molina and Rivera [18] and Junek [16]. It is also shown that, if $(\mathcal{A}, \alpha)$ is a normed ideal of operators in the sense of Pietsch [21], $E$ is an (FG)-space and $G$ is a (DFG)-space, then $\mathcal{A}^{w}(E, F)$ is a (DF)-space, where $\mathcal{A}^{w}$ denotes the largest (weak) extension of $\mathcal{A}$. The classes of (FG) and (DFG)-spaces are large as was shown in [5], [6], [9]. Concrete examples are mentioned after Definition 1. Some consequences on the distinguishedness of $E \hat{\otimes}_{\alpha} F$ and the barrelledness of $G \hat{\otimes}_{\alpha} H$ for $E, F$ Fréchet spaces and $G, H$ (DF)-spaces are also indicated.

Some of the results here are contained in the Doctoral Thesis of the first author written under the guidance of $J$. Bonet.

Our notation is standard, we refer to [15], [17] and [19]. If $E$ is a locally convex space (l.c.s.), $\mathcal{U}_{0}(E)$ and $\mathcal{B}(E)$ stand for the families of all absolutely convex (abx.) 0 -neighbourhoods and abx. bounded sets in $E$ respectively. If $E$ is a Fréchet space, we will say that $\left(U_{n}\right)_{n \in N}$ is a 0 -basis in $E$ if it is a decreasing basis of abx. closed 0-neighbourhoods in $E$. The absolutely convex hull of a subset $A$ of $E$ is denoted by $\Gamma(A)$. If $A$ is an abx. subset of $E$, we denote by $p_{A}$ the Minkowski functional associated with $A$ and $E_{A}:=[A] /$ ker $p_{A}$ endowed with the norm induced by $p_{A}$. We mean by $E_{b}^{\prime}$ the strong dual of $E$.

If $E$ and $F$ are 1.c.s., we denote by $\mathcal{L}(E, F)$ the space of all linear mappings from $E$ into $F$ and $L(E, F)$ is the subspace of continuous linear mappings.

We recall the definitions of (FG)-spaces and (DFG)-spaces given by Bonet, Díaz and Taskinen [6]. The definitions given below are slightly different from the original ones. The reason for this modification is that 
these are less restrictive than the original definitions, all the known results for these classes remain valid (see [6, Lemma 6]) even with clearer proofs and, surprisingly, it is possible to obtain more stability properties. Since Bonet, Díaz and Taskinen will use in the future these new definitions for the (FG)-spaces and (DFG)-spaces (personal communication), it will not cause any confusion.

Definition 1. A Fréchet space $E$ is said to be an (FG)-space if there is a 0 -basis $\left(U_{n}\right)_{n \in \mathbb{N}}$ in $E$ such that for every sequence $\left(\alpha_{k}\right)_{k \in \mathbb{N}}$, $\alpha_{k} \geq 1(k \in \mathbb{N})$ there are a sequence of operators $\left(P_{k}\right)_{k \in \mathbb{N}}$ in $L(E, E)$ and $a$ bounded subset $B \subset E$ satisfying

(1) $x=\sum_{j \in \mathbb{N}} P_{j}(x), \forall x \in E$,

(2) $P_{n}\left(\alpha_{n} U_{n}\right) \subset B, n \in \mathbb{N}$.

A (DF)-space $(G, t)$ is said to be a (DFG)-space if there is an increasing fundamental sequence $\left(B_{k}\right)_{k \in \mathrm{N}}$ of closed abx. bounded sets in $G$ and there is a locally convex topology $s$ in $G$ weaker than $t$ such that $(G, t)$ has a basis of $s$-closed abx. O-neighbourhoods and, for every sequence $\left(\alpha_{k}\right)_{k \in \mathbb{N}}, 0<\alpha_{k} \leq 1$, there are a sequence of operators $\left(Q_{k}\right)_{k \in \mathbb{N}}$ in $L((G, t),(G, t))$ and an s-closed abx. 0-neighbourhood $U$ in $(G, t)$ such that

(1) $x=\sum_{j \in \mathrm{N}} Q_{j}(x), \forall x \in G$, where the series converges for the topology $s$,

(2) $U \subset Q_{k}^{-1}\left(\alpha_{k} B_{k}\right)$ for every $k \in \mathbb{N}$.

If the topology $s$ can be taken equal to $t$ in the definition we will say that $(G, t)$ is a strong (DFG)-space.

The following are examples of (FG)-spaces [6]:

(i) Banach spaces and countable products of Banach spaces.

(ii) Banach valued Köthe echelon spaces of order $p, 1 \leq p<\infty$ or $p=0, \lambda_{p}\left(A,\left(X_{i}\right)_{i \in \mathbb{N}}\right)$ with $X_{i}$ Banach $(i \in \mathbb{N})$.

(iii) Generalized Dubinsky echelon spaces with decreasing steps which are Montel.

(iv) The Fréchet spaces of measurable functions introduced by Reiher, $L_{\rho}(A)$ with absolutely continuous $\rho$. In particular the spaces $L_{p}\left(\left(\mu_{n}\right)_{n \in \mathbb{N}}\right)$ of Grothendieck, $1 \leq p<\infty$, where $\mu_{i}$ are $\sigma$-finite measures $(i \in \mathbb{N})$. 
(v) Fréchet Schwartz spaces with a finite dimensional decomposition and a continuous norm.

(vi) The Fréchet space of continuous functions $C A_{0}(X)$.

Moreover, it is easy to see that (with the above definition) complemented subspaces of (FG)-spaces are also (FG)-spaces, then every Fréchet Schwartz space with the bounded approximation property and a continuous norm is an (FG)-space (see [5]).

The following are examples of (DFG)-spaces [6]:

(i) Normed spaces and countable direct sums of normed spaces.

(ii) Strong duals of (FG)-spaces.

(iii) The inductive limit of continuous functions $\operatorname{ind}_{n} C\left(v_{n}\right)_{0}(X)$ and the projective hulls $C \bar{V}(X), C \bar{V}_{0}(X)$.

\section{TENSOR PRODUCTS OF FRÉCHET AND (DF)-SPACES}

We need some definitions and a technical lemma on tensor norms. We refer to [19] and [8] for the notations.

If $E$ and $F$ are 1.c.s. and $\alpha$ is a tensor norm, the topology of $E \otimes_{\alpha} F$ is given by the system of seminorms $\left(p_{U} \otimes_{\alpha} p_{V}\right)(z):=\alpha\left(\left(\Phi_{U} \otimes\right.\right.$ $\left.\left.\Phi_{V}\right)(z) ; E_{U}, F_{V}\right), z \in E \otimes F, U \in \mathcal{U}_{0}(E), V \in \mathcal{U}_{0}(F)$, where $\Phi_{U}: E \longrightarrow$ $E_{U}, \Phi_{V}: F \longrightarrow F_{V}$ are the canonical maps.

If $A$ and $B$ are abx. subsets of $E$ and $F$, respectively, we will denote by $\alpha(A, B):=\left\{x \in E \otimes_{\alpha} F /\left(p_{A} \otimes_{\alpha} p_{B}\right)(x) \leq 1\right\}=\{x \in[A] \otimes$ $\left.[B] / \alpha\left(\left(\Phi_{A} \otimes \Phi_{B}\right)(x) ; E_{A}, E_{B}\right) \leq 1\right\}$.

Definition 2. If $E$ and $F$ are l.c.s., $\alpha$ is a tensor norm and $B$ is a bounded subset of $E \hat{\bigotimes}_{\alpha} F$, we will say that $B$ is localizable if there are $C \in \mathcal{B}(E), D \in \mathcal{B}(F)$ such that $B \subset \overline{\alpha(C, D)}$.

This concept of "localization" of bounded sets in $\alpha$-tensor products coincides with the usual ones when $\alpha=\pi(\overline{\pi(C, D)}=\overline{\Gamma(C \otimes D)})$ or $\alpha=\alpha_{p, q}$, the Lapresté tensor product (see [18], [16]).

The following lemma is necessary to obtain our main result then

Lemma 3.([20]) If $E_{i}$ and $F_{i}$ are l.c.s., $T_{i} \in \mathcal{L}\left(E_{i}, F_{i}\right), i=1,2$; 


$$
\left(T_{1} \otimes T_{2}\right)(\alpha(A, B)) \subset \alpha\left(T_{1}(A), T_{2}(B)\right)
$$

for every $A \subset E_{1}, B \subset E_{2}$ absolutely convex.

The following proposition improves $[6$, Corollary 4.6$]$ and $[18$, Theorem 2].

Proposition 4. If $E$ and $F$ are (FG)-spaces and $\alpha$ is a tensor norm, then the bounded sets of $E \hat{\otimes}_{\alpha} F$ are localizable and $E \hat{\otimes}_{\alpha} F$ is an (FG)-space.

Proof. By hypothesis, there are $\left(U_{n}\right)_{n \in \mathbb{N}}$ and $\left(V_{n}\right)_{n \in \mathbb{N}} 0$-basis in $E$ and $F$, respectively, such that for every sequence $\left(\lambda_{k}\right)_{k \in \mathbb{N}}, 1 \leq \lambda_{k}<\infty$ $(k \in \mathbb{N})$, there are $B \in \mathcal{B}(E), C \in \mathcal{B}(F), Q_{i} \in L(E, E)$, and $R_{i} \in$ $L(F, F), i \in \mathbf{N}$, satisfying:

(a) $\forall i \in \mathbb{N}$

$$
Q_{i}\left(\lambda_{i} U_{i}\right) \subset \frac{1}{2^{i}} B, \quad R_{i}\left(\lambda_{i} V_{i}\right) \subset \frac{1}{2^{i}} C,
$$

(b)

$$
\sum_{1}^{\infty} Q_{i}=I_{E} ; \quad \sum_{1}^{\infty} R_{i}=I_{F} \quad \text { pointwisely. }
$$

Defining $P_{i}:=\sum_{j=1}^{i} Q_{i} \otimes R_{j}+\sum_{j=1}^{i-1} Q_{j} \otimes R_{i}, i \in \mathbb{N}$, we easily obtain $\sum_{1}^{m} P_{i}=\left(\sum_{1}^{m} Q_{i}\right) \otimes\left(\sum_{1}^{m} R_{i}\right)$ and the sum converges pointwisely to $I_{E \otimes F}$ when $m$ tends to infinity. On the other hand, the equicontinuity of $\left(P_{i}\right)_{i \in \mathrm{N}}$ follows from the equicontinuity of $\left(Q_{i}\right)_{i \in \mathrm{N}}$ and $\left(R_{i}\right)_{i \in \mathbf{N}}$, and this implies that $\sum_{1}^{\infty} \hat{P}_{i}$ converges pointwisely to $I_{E \hat{Q}_{\alpha} F}$, where $P_{i}$ is the extension to $E \hat{\otimes}_{\alpha} F$ of $P_{i}, i \in \mathbb{N}$.

Setting $H:=\overline{\alpha(B, C)}^{E \hat{\otimes}_{\alpha} F}$, we obtain

$$
\begin{aligned}
\hat{P}_{i}\left(\lambda_{i} \alpha\left(U_{i}, V_{i}\right)\right) \subset & \left(\sum_{j=1}^{i} Q_{i} \hat{\otimes} R_{j}\right)\left(\alpha\left(\lambda_{i} U_{i}, \lambda_{j} V_{j}\right)\right) \\
& +\left(\sum_{j=1}^{i-1} Q_{j} \hat{\otimes} R_{i}\right)\left(\alpha\left(\lambda_{j} U_{j}, \lambda_{i} V_{i}\right)\right)
\end{aligned}
$$




$$
\subset \frac{1}{2^{i}} \sum_{j=1}^{i} \frac{1}{2^{j}} H+\frac{1}{2^{i}} \sum_{j=1}^{i-1} \frac{1}{2^{j}} H \subset \frac{1}{2^{i-1}} H,
$$

$i=1, \ldots, \infty$. Therefore $E \hat{\otimes}_{\alpha} F$ is an (FG)-space and, moreover, if $x \in \bigcap_{1}^{\infty} \lambda_{i}{\overline{\alpha\left(U_{i}, V_{i}\right)}}^{E \hat{\theta}_{\alpha} F}$, then

$$
\left(\sum_{1}^{m} \hat{P}_{i}\right)(x) \in\left(\sum_{1}^{m} \frac{1}{2^{i-1}}\right) H .
$$

Thus, since $x=\lim _{m \rightarrow \infty} \sum_{1}^{m} \hat{P}_{i}(x) \in 2 H$,

$$
\bigcap_{1}^{\infty} \lambda_{i}{\overline{\alpha\left(U_{i}, V_{i}\right)}}^{E \dot{\otimes}_{\alpha} F} \subset 2 H,
$$

which implies that the bounded sets of $E \hat{\otimes}_{\alpha} F$ are localizable.

The following technical lemma is essential to show that $G \otimes_{\alpha} H$ and $\mathcal{A}^{w}(E, G)$ are (DF)-spaces whenever $E$ is an (FG)-space and $G, H$ are (DFG)-spaces. It provides a useful property which is very close to (DFop) introduced in [7].

Lemma 5. Let $E$ be an (FG)-space and $G$ a (DFG)-space. There are two sequences: $\left(V_{n}\right)_{n \in \mathbf{N}}$ a O-basis in $E$ and $\left(B_{n}\right)_{n \in \mathbf{N}}$ a fundamental sequence of bounded sets in $G$ such that

(a) For every sequences $\left(\epsilon_{k}\right)_{k \in N}$ of strictly positive escalars and $\left(C_{n}\right)_{n \in \mathrm{N}}$ of bounded sets in $E$, there are $C \in \mathcal{B}(E)$ and $\left(Q_{k}\right)_{k \in \mathbb{N}} \subset$ $L(E, E)$ such that

$$
Q_{k}\left(V_{k}\right) \subset \epsilon_{k} C, \quad\left(I-\sum_{k=1}^{n} Q_{k}\right)\left(C_{n}\right) \subset C, \quad k, n \in \mathbb{N} .
$$

(b) For every sequences $\left(\epsilon_{k}\right)_{k \in \mathrm{N}}$ of strictly positive escalars and $\left(U_{n}\right)_{n \in \mathbb{N}}$ of 0 -neighbourhoods in $G$, there are $U \in \mathcal{U}_{0}(G)$ and $\left(R_{k}\right)_{k \in \mathbb{N}} \subset$ $L(G, G)$ such that

$$
R_{k}(U) \subset \epsilon_{k} B_{k}, \quad\left(I-\sum_{k=1}^{n} R_{k}\right)(U) \subset U_{n}, \quad k, n \in \mathbb{N} .
$$


Proof. We will show (b). The proof of (a) is analogous.

Since $(G, t)$ is a (DFG)-space, it has a fundamental sequence $\left(B_{n}\right)_{n \in \mathbb{N}}$ of absolutely convex bounded subsets and there is a locally convex topology $s$ on $G$, coarser than $t$, such that $(G, t)$ has a basis of $s$-closed absolutely convex 0 -neighbourhoods and for every sequence $\left(\delta_{k}\right)_{k \in \mathrm{N}}$ of strictly positive escalars there is a sequence $\left(R_{k}\right)_{k \in \mathrm{N}} C$ $L(G, G)$ such that

(i) $x=\sum_{k \in \mathrm{N}} R_{k}(x) \forall x \in G$, where the series converges for the topology $s$,

(ii) $\bigcap_{k \in N} R_{k}^{-1}\left(\delta_{k} B_{k}\right) \in \mathcal{U}_{0}(G)$.

Given two sequences: $\left(\epsilon_{k}\right)_{k \in \mathbb{N}}$ of strictly positive numbers and $\left(U_{n}\right)_{n \in \mathbb{N}}$ of 0 -neighbourhoods in $G$ (which we can suppose $s$-closed), we can find another sequence $\left(\delta_{k}\right)_{k \in \mathbb{N}}$ such that $0<\delta_{k}<\epsilon_{k}, k \in \mathbb{N}$, and $\sum_{k=n}^{m} \delta_{k} B_{k} \subset U_{n}, n \in \mathbb{N}, m \geq n$. Now choose $\left(R_{k}\right)_{k \in \mathbb{N}}$ satisfying (i) and (ii), and define $U:=\bigcap_{k \in \mathbb{N}} R_{k}^{-1}\left(\delta_{k} B_{k}\right)$. Then we have

$$
x-\sum_{k=1}^{n} R_{k}(x)=s-\lim _{m \rightarrow \infty} \sum_{k=n+1}^{m} R_{k}(x) \in U_{n},
$$

for all $x \in U$, since $U_{n}$ is s-closed, $n \in \mathbb{N}$.

We also need an easy but key lemma due to J. Bonet and the first author (see e.g. [11, Theorem 5.8]). A similar argument can be found in $[15]$.

Lemma 6. Let $E$ be a l.c.s. and $\left(B_{n}\right)_{n \in N}$ a sequence of absolutely convex bounded sets in $E$ with $2 B_{n} \subset B_{n+1}, n \in \mathbb{N}$, which satisfies the following property:

For every sequence $\left(U_{n}\right)_{n \in \mathrm{N}}$ of 0-neighbourhoods in $E$ there is $U \in$ $\mathcal{U}_{0}(E)$ such that

$$
U \subset \bigcap_{n=1}^{\infty}\left(U_{n}+B_{n}\right)
$$

Then $\left(\bar{B}_{n}\right)_{n \in \mathbb{N}}$ is a fundamental sequence of bounded sets in $E$.

Proof. Let us suppose that there is $B \in \mathcal{B}(E)$ such that $B \not \subset n \bar{B}_{n}$, $\forall n \in \mathbb{N}$. Then we can find a bounded sequence $\left(z_{n}\right)_{n \in \mathbb{N}}$ in $E$ with 
$z_{n} \notin n \bar{B}_{n}$ and, hence, there exists $U_{n} \in \mathcal{U}_{0}(E), n \in \mathbb{N}$, such that $z_{n} \notin n\left(B_{n}+U_{n}\right)$. But $\bigcap_{n \in \mathbb{N}}\left(B_{n}+U_{n}\right)$ is a neighbourhood of 0 in $E$, which contradicts the fact that $\left(z_{n}\right)_{n \in \mathrm{N}}$ is bounded.

Thus there is $n_{0} \in \mathbb{N}$ with $B \subset n_{0} \bar{B}_{n_{0}} \subset \bar{B}_{2 n_{0}}$.

From this lemma and the characterization of (DF)-spaces given by Bierstedt and Bonet in [3,5.A.Lemma], we deduce (see $[11,5.8]$ ) that a l.c.s. $E$ is a (DF)-space if and only if it has a sequence $\left(B_{n}\right)_{n \in \mathbb{N}}$ of absolutely convex bounded subsets such that for every sequences $\left(\epsilon_{k}\right)_{k \in \mathrm{N}}$ of strictly positive numbers and $\left(U_{n}\right)_{n \in \mathrm{N}}$ of absolutely convex 0 -neighbourhoods, there is $U \in \mathcal{U}_{0}(E)$ such that

$$
U \subset \bigcap_{n \in \mathbb{N}}\left(\sum_{k=1}^{n} \epsilon_{k} B_{k}+U_{n}\right) .
$$

This will be useful in the following

Proposition 7. If $\alpha$ is a tensor norm and $G, H$ are (DFG)-spaces (respectively, strong (DFG)-spaces) then $G \otimes_{\alpha} H$ is a (DF)-space (resp., a strong (DFG)-space).

Proof. Let $\left(B_{n}\right)_{n \in \mathbb{N}}$ and $\left(C_{n}\right)_{n \in \mathbb{N}}$ be the fundamental sequences of bounded sets in $G$ and $H$, respectively, which satisfy the property (DFG).

Given a sequence of strictly positive escalars $\left(\epsilon_{k}\right)_{k \in \mathbf{N}}\left(\epsilon_{k}<1, k \in\right.$ $\mathbb{N})$ and a sequence $\left(W_{n}\right)_{n \in \mathbb{N}}$ of 0 -neighbourhoods in $G \otimes_{\alpha} H$, find two decreasing sequences $\left(U_{n}\right)_{n \in \mathrm{N}}$ and $\left(V_{n}\right)_{n \in \mathrm{N}}$ of 0 -neighbourhoods in $G$ and $H$, respectively, such that

$$
\begin{aligned}
\text { (i) } & \alpha\left(U_{n}, V_{n}\right) \subset W_{n}, \\
\text { (ii) } & \alpha\left(\epsilon_{j} B_{j}, V_{n}\right) \subset W_{n}, \\
\text { (iii) } & \alpha\left(U_{n}, \epsilon_{j} C_{j}\right) \subset W_{n},
\end{aligned}
$$

$n \in \mathbb{N}, j=1 \ldots n$. By Lemma 5 , there are two sequences $\left(Q_{k}\right)_{k \in \mathbb{N}} \subset$ $L(G, G)$ and $\left(R_{k}\right)_{k \in \mathrm{N}} \subset L(H, H)$ and there are $U \in \mathcal{U}_{0}(G)$ and $V \in$ $\mathcal{U}_{0}(H)$ such that 


$$
\begin{gathered}
(j) \quad Q_{k}(U) \subset \frac{\epsilon_{k}}{2^{k}} B_{k} ; \quad R_{k}(V) \subset \frac{\epsilon_{k}}{2^{k}} C_{k}, \\
(j j) \quad\left(I-\sum_{k=1}^{n} Q_{k}\right)(U) \subset \frac{1}{3} U_{n} ; \quad\left(I-\sum_{k=1}^{n} R_{k}\right)(V) \subset \frac{1}{3} V_{n},
\end{gathered}
$$

$n, k \in \mathbb{N}$. Now we claim that

$$
\alpha(U, V) \subset \bigcap_{n \in \mathbb{N}}\left(\sum_{k=1}^{n} \epsilon_{k} \alpha\left(B_{k}, C_{k}\right)+W_{n}\right)
$$

To show this, take $x \in \alpha(U, V)$. Given $n \in \mathbb{N}$, define

$$
x_{k}:=\left(\sum_{j=1}^{k} Q_{k} \otimes R_{j}+\sum_{j=1}^{k-1} Q_{j} \otimes R_{k}\right)(x), \quad k=1 \ldots n .
$$

Since $Q_{k}(U) \subset \frac{\epsilon_{k}}{2^{k}} B_{k}$ and $R_{j}(V) \subset \frac{\epsilon_{j}}{2^{j}} C_{j}$, we have that $\left(Q_{k} \otimes R_{j}\right)(x) \in$ $\frac{\epsilon_{k} \epsilon_{j}}{2^{k+j}} \alpha\left(B_{k}, C_{j}\right), j, k \in \mathbb{N}$. Hence

$$
\begin{gathered}
x_{k} \in \sum_{j=1}^{k} \frac{\epsilon_{k}}{2^{1+j}} \alpha\left(B_{k}, C_{j}\right)+\sum_{j=1}^{k-1} \frac{\epsilon_{k}}{2^{1+j}} \alpha\left(B_{j}, C_{k}\right) \\
\subset \epsilon_{k}\left(\sum_{j=1}^{k} \frac{1}{2^{1+j}} \alpha\left(B_{k}, C_{j}\right)+\sum_{j=1}^{k-1} \frac{1}{2^{1+j}} \alpha\left(B_{j}, C_{k}\right)\right) \subset \epsilon_{k} \alpha\left(B_{k}, C_{k}\right),
\end{gathered}
$$

$k=1 \ldots n$. Define now $y_{n}:=x-\sum_{k=1}^{n} x_{k}$. It easily follows that

$$
y_{n}=\left(\sum_{j=1}^{n}\left(I-\sum_{k=1}^{n} Q_{k}\right) \otimes R_{j}\right)(x)+\left(\sum_{j=1}^{n} Q_{j} \otimes\left(I-\sum_{k=1}^{n} R_{k}\right)\right)
$$




$$
+\left(\left(I-\sum_{k=1}^{n} Q_{k}\right) \otimes\left(I-\sum_{k=1}^{n} R_{k}\right)\right)(x) .
$$

Finally, from (i), (ii), (iii), $(j)$ and $(j j)$, we obtain

(1) $\left(\left(I-\sum_{k=1}^{n} Q_{k}\right) \otimes R_{j}\right)(x) \in \frac{1}{3} \cdot \frac{1}{2^{j}} \alpha\left(U_{n}, \epsilon_{j} C_{j}\right) \subset \frac{1}{3} \cdot \frac{1}{2^{j}} W_{n}$,

(2) $\left(Q_{j} \otimes\left(I-\sum_{k=1}^{n} R_{k}\right)\right)(x) \in \frac{1}{3} \cdot \frac{1}{2^{j}} \alpha\left(\epsilon_{j} B_{j}, V_{n}\right) \subset \frac{1}{3} \cdot \frac{1}{2^{j}} W_{n}$,

(3) $\left(\left(I-\sum_{k=1}^{n} Q_{k}\right) \otimes\left(I-\sum_{k=1}^{n} R_{k}\right)\right)(x) \in \frac{1}{9} \alpha\left(U_{n}, V_{n}\right) \subset \frac{1}{3} W_{n}$,

$j=1 \ldots n$, which implies that $y_{n} \in W_{n}$. This shows that $G \otimes_{\alpha} H$ is a (DF)-space by lemma 6 .

If $G$ and $H$ are strong (DFG)-spaces, to prove that $G \otimes_{\alpha} H$ is a strong (DFG)-space, it remains to show that $\lim _{n} \sum_{k=1}^{n} x_{k}=x$. But this is a consequence of the fact that $\left(\sum_{k=1}^{n} Q_{k}\right)_{n \in \mathrm{N}}$ and $\left(\sum_{k=1}^{n} R_{k}\right)_{n \in \mathrm{N}}$ converge pointwisely to the identity of $G$ and $H$, respectively.

Remarks: (1) For finitely generated tensor norms $\alpha$, it is possible to give a shorter and more elegant argument to show that $G \otimes_{\alpha} H$ is a (DF)-space whenever $G$ and $H$ are (DFG)-spaces. The proof was kindly provided by Andreas Defant: By [8, 29.2, 29.7, 29.8 and 35.3], there is a Banach space $X$ and a surjective topological homomorphism

$$
\Psi:\left(G \otimes_{\varepsilon} X^{*}\right) \otimes_{\pi}\left(X \otimes_{\alpha} H\right) \longrightarrow G \otimes_{\alpha} H .
$$

Since $G$ is a (DFG)-space, the space $G \otimes_{\epsilon} X^{*}$ is a (DF)-space (see [6, Proposition 9]). By the same argument, $H \otimes_{\alpha} X$ is a quotient of $\left(H \otimes_{\varepsilon} X^{*}\right) \otimes_{\pi}\left(X \otimes_{\alpha} X\right)$, which is a (DF)-space since $H$ is a (DFG)-space 
and the projective tensor product of two (DF)-spaces is a (DF)-space. This implies that $G \otimes_{\alpha} H$ is also a (DF)-space.

(2) Note that, by Lemma 6, we have shown in the above proposition that the bounded subsets of $G \otimes_{\alpha} H$ are localizable if $G$ and $H$ are (DFG)-spaces.

In order to obtain more properties, such as distinguishedness for $\alpha$ tensor products of Fréchet spaces or barrelledness for $\alpha$-tensor products of (DF)-spaces, we need some definitions and notation.

The density condition, (DC), was introduced by Heinrich [14] and it was thoroughly studied by Bierstedt and Bonet (see [1], [2], [3], [4]) in the context of Fréchet spaces. A Fréchet space $E$ has the density condition if and only if the bounded sets of $E_{b}^{\prime}$ are metrizable [1]. Quasinormable spaces and Fréchet Montel spaces have the (DC). Every Fréchet space $E$ with (DC) is distinguished, that is $E_{b}^{\prime}$ is bornological. Bierstedt and Bonet also introduced the dual density condition, (DDC), (see [2]). A (DF)-space $G$ has the (DDC) if and only if its bounded sets are metrizable, and every (DF)-space with (DDC) is quasibarrelled (see [2]).

The (DC) and (DDC) are stable under projective and injective tensor products in the context of (FG)-spaces and barrelled strong (DFG)spaces, respectively (see [4], [9]). Our purpose is to generalize these results for arbitrary tensor norms. We need the following

Definition 8. Let $E$ be a Fréchet space and $\left(U_{n}\right)_{n \in \mathrm{N}}$ a 0 -basis in $E$. $E$ is said to satisfy the density condition by operators, briefly (DCo), if there is $B \in \mathcal{B}(E)$ such that for all $m \in \mathbb{N}$ and $B^{\prime} \in \mathcal{B}(E)$, there exist $\lambda>0$ and $P \in L(E, E)$ such that

(i) $P\left(B^{\prime}\right) \subset U_{m}$,

(ii) $(I-P)\left(B^{\prime}\right) \subset \lambda B$.

Let $G$ be a (DF)-space and $\left(B_{n}\right)_{n \in \mathbb{N}}$ a fundamental sequence of bounded sets in $G$. $G$ is said to satisfy the dual density condition by operators, briefly (DDCo), if there is $U \in \mathcal{U}_{0}(G)$ such that for all $m \in \mathbb{N}$ and $V \in \mathcal{U}_{0}(G)$, there are $\varepsilon>0$ and $P \in L(G, G)$ such that

(i) $P\left(B_{m}\right) \subset V$,

(ii) $(I-P)(\varepsilon U) \subset V$. 
Remark: It easily follows from these definitions and the characterizations of (DC) and (DDC) due to Bierstedt and Bonet in the context of Fréchet spaces and (DF)-spaces, that every Fréchet space $E$ (respectively, (DF)-space $G$ ) with (DCo) (resp., (DDCo)) satisfies the (DC) (resp., (DDC)).

Proposition 9. Every (FG)-space with the (DC) satisfies property (DCo).

Proof. Let $E$ be an (FG)-space with a 0-basis $\left(U_{n}\right)_{n \in N}$ and $\left(P_{n}\right)_{n \in \mathbb{N}}$ any sequence of bounded operators in $E$ associated to some sequence of strictly positive scalars by property (FG). Then, as a consequence of [10], $\left(P_{n}\right)_{n \in \mathbb{N}}$ is a resolution of the identity of $E$ with property (M) of Díaz and Miñarro, that is, for every $B \in \mathcal{B}(E)$ and every $U \in \mathcal{U}_{0}(E)$, we have

$$
\lim _{n \rightarrow \infty} \sup _{x \in B} p_{U}\left(\sum_{j=n}^{\infty} p_{j}(x)\right)=0 .
$$

Now, since $\left(P_{n}\right)_{n \in \mathbf{N}}$ is a sequence of bounded operators, we can find $B \in B(E)$ such that $P_{n}^{-1}(B) \in \mathcal{U}_{0}(E), \forall n \in \mathbb{N}$. Let $m \in \mathbb{N}$ and $B^{\prime} \in \mathcal{B}(E)$. Then, by property $(\mathrm{M})$, there is $n_{0} \in \mathbb{N}$ such that, for $P:=\sum_{n_{0}+1}^{\infty} P_{n}$, the following is satisfied

$$
P\left(B^{\prime}\right)=\sum_{n_{0}+1}^{\infty} P_{n}\left(B^{\prime}\right) \subset U_{m}
$$

Moreover,

$$
(I-P)^{-1}(B)=\left(\sum_{1}^{n_{0}} P_{n}\right)^{-1}(B) \in \mathcal{U}_{0}(E)
$$

which yields the result.

Proposition 10. If $G$ is a barrelled strong (DFG)-space with (DDC), then it satisfies (DDCo).

Proof. Let $\left(B_{n}\right)_{n \in N}$ be a fundamental sequence of bounded subsets in $G$ and $\left(P_{n}\right)_{n \in \mathbb{N}}$ any sequence of bounded operators in $G$ associated 
to some sequence of strictly positive scalars by property (DFG). Then, as a consequence of [9], $\left(P_{n}\right)_{n \in \mathrm{N}}$ is a resolution of the identity of $G$ with property (M).

Now, since $\left(P_{n}\right)_{n \in \mathrm{N}}$ is a sequence of bounded operators, we can find $U \in \mathcal{U}_{0}(G)$ such that $P_{n}(U) \in \mathcal{B}(G), \forall n \in \mathbb{N}$. Let $m \in \mathbb{N}$ and $V \in \mathcal{U}_{0}(G)$. Then, by property (M), there is $n_{0} \in \mathbf{N}$ such that, for $P:=\sum_{1}^{n_{0}} P_{n}$, the following is satisfied

$$
(I-P)\left(B_{m}\right)=\sum_{n_{0}+1}^{\infty} P_{n}\left(B_{m}\right) \subset V .
$$

On the other hand,

$$
P(U)=\sum_{1}^{n_{0}} P_{n}(U) \in \mathcal{B}(G),
$$

and we obtain the result.

Díaz, López Molina and Rivera showed in [9] that the strong duals of (FG)-spaces with (DC) are examples of barrelled strong (DFG)-spaces with (DDC).

Proposition 11. If $\alpha$ is a tensor norm, $E$ and $F$ are Fréchet spaces (respectively, (DF)-spaces) with the (DCo) (resp., (DDCo)) and the bounded subsets of $E \hat{\otimes}_{\alpha} F$ are localizable, then $E \hat{\otimes}_{\alpha} F$ also satisfies (DCo) (resp., (DDCo)).

Proof. We will show the result for (DCo). The proof for (DDCo) is analogous.

Let $\left(U_{k}\right)_{k \in \mathbf{N}}$ and $\left(V_{k}\right)_{k \in \mathbf{N}}$ 0-basis in $E$ and $F$, respectively. If $E$ and $F$ satisfy (DCo), then there are $B \in \mathcal{B}(E)$ and $C \in \mathcal{B}(F)$ such that for all $m, m^{\prime} \in \mathbb{N}$ and for all $B^{\prime} \in \mathcal{B}(E), C^{\prime} \in B(F)$, there are $\lambda, \lambda^{\prime}>0$ and $P \in L(E, E), Q \in L(F, F)$ which satisfy

(i) $P\left(B^{\prime}\right) \subset U_{m}, \quad(I-P)\left(B^{\prime}\right) \subset \lambda B$.

(ii) $Q\left(C^{\prime}\right) \subset V_{m^{\prime}}, \quad(I-Q)\left(C^{\prime}\right) \subset \lambda C$.

Let $n \in \mathbb{N}, B^{\prime} \in \mathcal{B}(E)$ and $C^{\prime} \in \mathcal{B}(F)$. Find $m \in \mathbb{N}(m \geq n)$ with $2 U_{m} \subset \varepsilon U_{n}$, where $\varepsilon>0$ satisfies $\varepsilon C^{\prime} \subset V_{n}$, and find $m^{\prime} \in \mathbb{N}\left(m^{\prime} \geq n\right)$ with $2 V_{m^{\prime}} \subset \varepsilon^{\prime} V_{n}$, where $\varepsilon^{\prime}>0$ satisfies $\varepsilon^{\prime} \lambda B \subset U_{n}$. Then we have 
(a)

$$
[(I-P) \otimes(I-Q)]\left(\alpha\left(B^{\prime}, C^{\prime}\right)\right) \subset \alpha\left(U_{m}, V_{m^{\prime}}\right) \subset \alpha\left(U_{n}, V_{n}\right)
$$

(b)

$$
\begin{gathered}
{[I-(I-P) \otimes(I-Q)]\left(\alpha\left(B^{\prime}, C^{\prime}\right)\right)=[(I-P) \otimes Q+P \otimes I]\left(\alpha\left(B^{\prime}, C^{\prime}\right)\right) \subset} \\
\alpha\left(\lambda B, V_{m^{\prime}}\right)+\alpha\left(U_{m}, C^{\prime}\right) \subset \frac{1}{2} \alpha\left(U_{n}, V_{n}\right)+\frac{1}{2} \alpha\left(U_{n}, V_{n}\right)=\alpha\left(U_{n}, V_{n}\right) .
\end{gathered}
$$

From this we conclude that $E \hat{\otimes}_{\alpha} F$ satisfies (DCo).

Corollary 12. If $\alpha$ is a tensor norm and $E, F$ are (FG)-spaces with the (DC) (respectively, barrelled strong (DFG)-spaces with the (DDC)), then $E \hat{\otimes}_{\alpha} F$ is a Fréchet space with $(D C)$, hence distinguished (respectively, a barrelled (DF)-space with (DDC)).

It is worth to mention that the Fréchet spaces quasinormable by operators (respectively, the (DF)-spaces with property $(Q N o)$ ), introduced and studied in [20], are examples of Fréchet spaces with (DCo) (resp., (DF)-spaces with (DDCo)).

\section{IDEALS OF OPERATORS AND (DF)-SPACES}

Following Piestch [21], the following properties define a normed ideal $(\mathcal{A}, \alpha)$ of operators on the class of Banach spaces:

(I1) $\mathcal{F}(X, Y) \subset \mathcal{A}(X, Y) \subset L(X, Y)$, for all Banach spaces $X, Y$, where $\mathcal{F}(X, Y)$ denotes the space of all finite rank operators.

(I2) If $S \in \mathcal{A}(X, Y), T \in L\left(Z_{1}, X\right)$ and $R \in L\left(Y, Z_{2}\right)$, then $R \circ S \circ$ $T \in \mathcal{A}\left(Z_{1}, Z_{2}\right)$.

(I3) $\alpha$ is a complete norm on each component $\mathcal{A}(X, Y)$ satisfying $\alpha\left(x^{\prime} \otimes y\right)=\left\|x^{\prime}\right\|\|y\|, x^{\prime} \in X^{*}, y \in Y$, and $\alpha(R \circ S \circ T) \leq\|R\|\|T\| \alpha(S)$ for all operators $R, S, T$ as in (I2).

Among the several possibilities to extend an ideal $\mathcal{A}$ of operators on Banach spaces to the category of locally convex spaces, we will concentrate on the largest extension $\mathcal{A}^{w}$ (see [21]). More precisely, if $E$ and $F$ are locally convex spaces ( $E$ quasi-complete) and $S \in L(E, F)$, 
then $S \in \mathcal{A}^{w}(E, F)$ if, and only if, for all Banach spaces $X, Y$ and for all operators $T \in L(X, E), R \in L(F, Y)$ the product $R \circ S \circ T$ belongs to $\mathcal{A}(X, Y)$. That is, for all $B \in \mathcal{B}(E)$ and $U \in \mathcal{U}_{0}(F)$ the operator $\Phi_{U} \circ S \circ \Phi_{B}$ belongs to $L\left(\hat{E_{B}}, \hat{F_{U}}\right)$, where $\Phi_{B}: \hat{E_{B}} \rightarrow E$ and $\Phi_{U}: F \rightarrow \hat{F}_{U}$ are the canonical mappings.

The space $\mathcal{A}^{w}(E, F)$ admits a natural topology induced by proj $\mathcal{A}\left(\hat{E}_{B}, \hat{F}_{U}\right)$. In order to give a description of a basis of 0 -neighbourhoods, let us establish the following notation: If $A \subset E, B \subset F$ are absolutely convex and $S \in L(E, F)$ satisfies $S(A) \subset B$, we denote by $S_{A, B}: \hat{E_{A}} \rightarrow \hat{F_{B}}$ the induced map. Now we write

$$
\begin{gathered}
\mathcal{W}(A, B):=\left\{S \in \mathcal{A}^{w}(E, F) / S(A) \subset B, S_{A, B} \in \mathcal{A}\left(\hat{\left.E_{A}, \hat{F}_{B}\right)}\right.\right. \\
\text { and } \left.\alpha\left(S_{A, B}\right) \leq 1\right\} .
\end{gathered}
$$

With this notation, the family $\left\{\mathcal{W}(B, U) / B \in \mathcal{B}(E), U \in \mathcal{U}_{0}(F)\right\}$ is a basis of 0 -neighbourhoods in $\mathcal{A}^{w}(E, F)$.

For instance, if $\mathcal{A}$ is the ideal of all bounded operators and $\alpha$ is the operator norm, then $\mathcal{A}^{w}(E, F)$ is the space $L(E, F)$ of all continuous linear operators endowed with the topology of uniform convergence on the bounded subsets of $E$. And, for $\mathcal{A}=\mathcal{K}$ the ideal of compact operators, $\mathcal{K}^{\psi}(E, F)$ coincides with the space of Montel operators from $E$ into $F$, that is, the operators that send each bounded subset of $E$ into a precompact set in $F$.

Remark: As a consequence of (I2) and (I3), it is easily seen that, for $A_{1}, A_{2} \subset E$ and $B_{1}, B_{2} \subset F$ absolutely convex, $S \in \mathcal{W}\left(A_{2}, B_{1}\right)$ and $T \in L(E, E), R \in L(F, F)$ satisfying $T\left(A_{1}\right) \subset A_{2}$ and $R\left(B_{1}\right) \subset B_{2}$, the product $R \circ S \circ T$ belongs to $\mathcal{W}\left(A_{1}, B_{2}\right)$. This will be used later on.

The following theorem should be compared with [6, Theorem 4.5], where Bonet, Díaz and Taskinen show that, under the same hypothesis, the space $L_{b}(E, G)$ is a (DF)-space using a similar argument.

Theorem 13. Let $(\mathcal{A}, \alpha)$ be a normed ideal of operators in Banach spaces, $E$ an (FG)-space and $G$ a (DFG)-space. Then $\mathcal{A}^{\omega}(E, G)$ is a (DF)-space. 
Proof. Let $\left(V_{n}\right)_{n \in \mathbb{N}}$ and $\left(B_{n}\right)_{n \in \mathbb{N}}$ be the 0 -basis and fundamental sequence of bounded sets in $E$ and $G$ respectively, which satisfy the corresponding properties (FG) and (DFG).

Given a sequence of strictly positive escalars $\left(\epsilon_{k}\right)_{k \in \mathbb{N}}\left(\epsilon_{k}<1, k \in\right.$ $\mathbb{N})$ and a sequence $\left(W_{n}\right)_{n \in \mathbb{N}}$ of 0 -neighbourhoods in $\mathcal{A}^{\omega}(E, G)$, find a sequence $\left(C_{n}\right)_{n \in \mathrm{N}}$ of bounded sets in $E$ and a sequence $\left(U_{n}\right)_{n \in \mathbb{N}}$ of 0 -neighbourhoods in $G$ such that

$$
\begin{aligned}
\text { (i) } & \mathcal{W}\left(C_{n}, U_{n}\right) \subset W_{n}, \\
\text { (ii) } & \mathcal{W}\left(\epsilon_{j}^{-1} V_{j}, U_{n}\right) \subset W_{n}, \\
\text { (iii) } & \mathcal{W}\left(C_{n}, \epsilon_{j} B_{j}\right) \subset W_{n},
\end{aligned}
$$

$n \in \mathbb{N}, j=1 \ldots n$. By Lemma 5 , there are two sequences $\left(Q_{k}\right)_{k \in \mathbb{N}} \subset$ $L(E, E)$ and $\left(R_{k}\right)_{k \in \mathbb{N}} \subset L(G, G)$ and there are $C \in \mathcal{B}(E)$ and $U \in \mathcal{U}_{0}(G)$ such that

(j) $\quad Q_{k}\left(V_{k}\right) \subset \frac{\epsilon_{k}}{2^{k}} C_{;} \quad R_{k}(U) \subset \frac{\epsilon_{k}}{2^{k}} B_{k}$,

$$
\text { (jj) } \quad\left(I-\sum_{k=1}^{n} Q_{k}\right)\left(C_{n}\right) \subset \frac{1}{3} C ; \quad\left(I-\sum_{k=1}^{n} R_{k}\right)(U) \subset \frac{1}{3} U_{n},
$$

$n, k \in \mathbb{N}$. Now we claim that

$$
\mathcal{W}(C, U) \subset \bigcap_{n \in \mathbb{N}}\left(\sum_{k=1}^{n} \epsilon_{k} \mathcal{W}\left(V_{k}, B_{k}\right)+W_{n}\right)
$$

To show this, take $f \in \mathcal{W}(C, U)$. Given $n \in \mathbb{N}$, define

$$
f_{k}:=\sum_{j=1}^{k} R_{k} \circ f \circ Q_{j}+\sum_{j=1}^{k-1} R_{j} \circ f \circ Q_{k}, \quad k=1 \ldots n .
$$

Since $R_{k}(U) \subset \frac{\epsilon_{k}}{2^{k}} B_{k}$ and $Q_{j}\left(V_{j}\right) \subset \frac{\epsilon_{j}}{2^{2}} C$, we have that $R_{k} \circ f \circ Q_{j} \in$ $\frac{\epsilon_{k} \epsilon_{j}}{2^{k+j}} \mathcal{W}\left(V_{j}, B_{k}\right), j, k \in \mathbb{N}$. Hence

$$
f_{k} \in \sum_{j=1}^{k} \frac{\epsilon_{k}}{2^{1+j}} \mathcal{W}\left(V_{j}, B_{k}\right)+\sum_{j=1}^{k-1} \frac{\epsilon_{k}}{2^{1+j}} \mathcal{W}\left(V_{k}, B_{j}\right)
$$


$\subset \epsilon_{k}\left(\sum_{j=1}^{k} \frac{1}{2^{1+j}} \mathcal{W}\left(V_{j}, B_{k}\right)+\sum_{j=1}^{k-1} \frac{1}{2^{1+j}} \mathcal{W}\left(V_{k}, B_{j}\right)\right) \subset \epsilon_{k} \mathcal{W}\left(V_{k}, B_{k}\right)$

$k=1 \ldots n$. Define now $g_{n}:=f-\sum_{k=1}^{n} f_{k}$. It easily follows that

$$
\begin{gathered}
g_{n}=\sum_{j=1}^{n}\left(I-\sum_{k=1}^{n} R_{k}\right) \circ f \circ Q_{j}+\sum_{j=1}^{n} R_{j} \circ f \circ\left(I-\sum_{k=1}^{n} Q_{k}\right) \\
+\left(I-\sum_{k=1}^{n} R_{k}\right) \circ f \circ\left(I-\sum_{k=1}^{n} Q_{k}\right) .
\end{gathered}
$$

Finally, from $(i),(i i),(i i i),(j)$ and $(j j)$, we obtain

(1) $\left(I-\sum_{k=1}^{n} R_{k}\right) \circ f \circ Q_{j} \in \frac{1}{3} \cdot \frac{1}{2^{j}} \mathcal{W}\left(\epsilon_{j}^{-1} V_{j}, U_{n}\right) \subset \frac{1}{3} \cdot \frac{1}{2^{j}} W_{n}$,

(2) $R_{j} \circ f \circ\left(I-\sum_{k=1}^{n} Q_{k}\right) \in \frac{1}{3} \cdot \frac{1}{2^{j}} \mathcal{W}\left(C_{n}, \epsilon_{j} B_{j}\right) \subset \frac{1}{3} \cdot \frac{1}{2^{j}} W_{n}$

(3) $\left(I-\sum_{k=1}^{n} R_{k}\right) \circ f \circ\left(I-\sum_{k=1}^{n} Q_{k}\right) \in \frac{1}{9} \mathcal{W}\left(C_{n}, U_{n}\right) \subset \frac{1}{3} W_{n}$,

$j=1 \ldots n$, which implies that $g_{n} \in W_{n}$.

Remark: Given $(\dot{\mathcal{A}}, \alpha)$ a normed ideal of operators, there is another interesting extension of $\mathcal{A}$ to the category of locally convex spaces, the smallest (strong) extension $\mathcal{A}^{*}$ (see [21]): If $E, F$ are l.c.s. with $F$ quasicomplete and $S \in L(E, F)$, then $S \in \mathcal{A}^{s}(E, F)$ if, and only if, there is some factorization $S=R_{0} \circ S_{0} \circ T_{0}$, where $T_{0} \in L\left(E, X_{0}\right)$, $R_{0} \in L\left(Y_{0}, F\right)$ and $S_{0} \in \mathcal{A}\left(X_{0}, Y_{0}\right)$ for certain Banach spaces $X_{0}, Y_{0}$. The Banach spaces $X_{0}, Y_{0}$ can be choosen to be the canonical Banach spaces $\hat{E}_{U}, \hat{F}_{B}$ for some $U \in \mathcal{U}_{0}(E), B \in B(F)$. Since 


$$
\mathcal{A}^{s}(E, F)=\bigcup_{\substack{v \in \mathcal{U}_{0}(E) \\ B \in \mathcal{B}(F)}} \mathcal{A}\left(\hat{E_{U}}, \hat{F_{B}}\right),
$$

the natural topology on $\mathcal{A}^{s}(E, F)$ is the locally convex inductive topology ind $\mathcal{A}\left(\hat{E}_{U}, \hat{F}_{B}\right)$.

Obviously $\mathcal{A}^{s}(E, F)$ is continuously embeded in $\mathcal{A}^{w}(E, F)$. Now, following the proof of the above theorem, if $E$ is an (FG)-space and $G$ is a quasicomplete (DFG)-space, the bounded sets of $\mathcal{A}^{w}(E, G)$ are contained in the closure (in $\mathcal{A}^{w}(E, G)$ ) of the bounded subsets of $\mathcal{A}^{s}(E, G)$ by Lemma 6 . This can have consequences on the algebraic (or even topological) equality $\mathcal{A}^{s}(E, G)=\mathcal{A}^{w}(E, G)$.

Acknowledgement: We are indebted with J. Bonet for his valuable suggestions concerning the final version of this paper.

\section{References}

[1] Bierstedt, K.D. and Bonet, J., Stefan Heinrich's density condition for Fréchet spaces and the characterization of the distinguished Köthe echelon spaces, Math. Nachr. 135 (1988), 149-180.

[2] Bierstedt, K.D. and Bonet, J., Dual density conditions in (DF)-spaces $I$, Results in Math. 14 (1988), 242-274

[3] Bierstedt, K.D. and Bonet, J., Dual density conditions in (DF)-spaces II, Bull. Soc. Roy. Sci. Liège 57 (1988), 567-589.

[4] Bierstedt, K.D. and Bonet, J., Density condition in Fréchet and (DF)-spaces, Rev. Mat. Univ. Complutense Madrid, 2, no. suplementario, (1989), 59-76.

[5] Bonet, J. and Díaz, J.C., The problem of topologies of Grothendieck and the class of Fréchet T-spaces, Math. Nachr. 150 (1991), 109-118.

[6] Bonet, J., Díaz, J.C. and Taskinen, J., Tensor stable Fréchet spaces and (DF)-spaces, Collect. Math. 42, 3 (1991), 199-236.

[7] Boyd, C. and Peris, A., A projective description of the Nachbin-ported topology, J. Math. Anal. Appl. 197 (1996), 635-657. 
[8] Defant, A. and Floret, K., Tensor Norms and Operator Ideals, NorthHolland Math. Studies 176, North-Holland, Amsterdam, 1993.

[9] Díaz, J.C., López Molina, J.A. and Rivera, M.J., Distinguished injective tensor products of Fréchet spaces, Proc. Roy. Irish Acad. 92A, 2 (1992), 165-174.

[10] Díaz, J.C. and Miñarro, M.A., Distinguished Fréchet spaces and projective tensor product, DOGA Tr. Math. J. 14 (1990), 191-208.

[11] Frerick, L., On vector valued sequence spaces, Ph. D. Thesis, Trier, 1994.

[12] Grothendieck, A., Sur les espoces (F) et (DF), Summa Brasil Math. 3 (1954), 57-123.

[13] Grothendieck, A., Produits tensoriels topologiques et espaces nucléaines, Mem. Amer. Math. Soc. 16 (1955).

[14] Heinrich, S., Ultrapowers of locally convex spaces and applications I, Math. Nachr. 118 (1984), 285-315.

[15] Jarchow, H., Locally convex spaces, B.G. Teubner, Stuttgart 1981.

[16] Junek, H., Factorization of operator ideals and bounded sets in tensor products of locally convex spaces, In: Functional Analysis (Ed. by K.D. Bierstedt, A. Pietsch, W. Ruess and D. Vogt), 203-216. Marcel Dekker, New York 1993.

[17] Köthe, G., Topological vector spaces $I, I$, , Springer Verlag, Heidelberg $1969,1979$.

[18] López Molina, J.A. and Rivera, M.J., Localization of bounded sets in the tensor products of Lapresté, Math. Japonica 38, 4 (1993), 643-653.

[19] Pérez Carreras, P. and Bonet, J., Barrelled locally convex spaces, North-Holland Math. Studies 131, Amsterdam 1987.

[20] Peris, A., Quasinormable spaces and the problem of topologies of Grothendieck, Ann. Acad. Sci. Fenn., Ser. A I 19 (1994), 167-203.

[21] Pietsch, A., Operator ideals, Akademie-Verlag, Berlin 1978.

[22] Taskinen, J., Counterexamples to "problème des topologies" of Grothendieck, Ann. Acad. Sci. Fenn., Ser. A I Math. Dissertationes 63 (1986). 
[23] Taskinen, J., (FBa)- and (FBB)-spaces, Math. Z. 198 (1988), 339365 . 